\title{
Riscrittura e imitazione omerica ne L'Italia liberata dai Goti di Gian Giorgio Trissino
}

\section{Paola Pecci}

\section{(2) OpenEdition \\ Journals}

Édition électronique

URL : https://journals.openedition.org/eve/1232

DOI : 10.4000/eve.1232

ISSN : 2425-1593

Éditeur :

Université de Savoie, Université Jean Moulin - Lyon 3

Référence électronique

Paola Pecci, «Riscrittura e imitazione omerica ne L'Italia liberata dai Goti di Gian Giorgio Trissino », Corpus Eve [En ligne], 2 | 2015, mis en ligne le 31 décembre 2015, consulté le 28 avril 2022. URL: http://journals.openedition.org/eve/1232 ; DOI : https://doi.org/10.4000/eve.1232

Ce document a été généré automatiquement le 28 avril 2022

(c) Tous droits réservés 


\title{
Riscrittura e imitazione omerica ne L'Italia liberata dai Goti di Gian Giorgio Trissino
}

\author{
Paola Pecci
}

\section{NOTE DE L'AUTEUR}

L'annexe est également disponible sous forme d'un fichier PDF joint à cet article.

1 In poche opere del Rinascimento italiano l'imitatio omerica è così scoperta e palese quanto nell'Italia liberata dai Goti di Gian Giorgio Trissino, al punto da aver indotto alcuni studiosi a definire il riuso della fonte omerica da parte del Vicentino nei termini di culto, devozione, adorazione nei confronti dell'autore dell'Iliade ${ }^{1}$, quell'Omero che lo stesso Trissino, nella lettera dedicatoria a Carlo V che precede il suo poema, dice di aver assunto per «Duce» e per «Idea» durante la composizione dell'opera.

2 Se in molti hanno rilevato l'ingombrante presenza di Omero all'interno dell'Italia liberata - date anche le dichiarazioni di poetica dell'autore, che non lasciano adito a dubbi circa i suoi modelli ispiratori ${ }^{2}$ - in pochi tuttavia hanno indagato come quest'imitatio concretamente si esplichi all'interno del poema ${ }^{3}$. Propongo di seguito $i$ risultati della mia ricerca in tal senso ${ }^{4}$. Partendo dalla teoria poetica del Vicentino e attraverso un breve iter all'interno della sua opera magna, giungerò all'analisi di alcune carte manoscritte trissiniane, facenti parte del cosiddetto Fondo Castiglioni, oggi conservato presso la Biblioteca Nazionale Braidense di Milano: carte preziose, non solo in quanto permettono di entrare nel laboratorio di scrittura di Trissino, ma anche perché lasciano trapelare dati interessanti circa la qualità e le caratteristiche dell'imitazione omerica all'interno dell'Italia liberata dai Goti. 


\section{L'Iliade ne L'Italia liberata dai Goti: dalla teoria alla prassi poetica}

3 Già dalla Divisione VI della Poetica trissiniana - pubblicata postuma (1562), insieme alla quinta, ma terminata dall'autore pochi mesi prima della morte ${ }^{5}$ - emerge con forza la devozione del poeta vicentino nei confronti di Omero. In questa sezione del suo trattato dedicata al poema eroico - e fedelissima al dettato aristotelico, al punto da rivelarsi quasi una traduzione dell'antica Poetica greca - Trissino addita Omero quale supremo modello da imitare, quale autore «più d'ogni altro meraviglioso» ${ }^{6}$, degno, assai più di Virgilio, di essere eletto a suo maestro e guida ispiratrice durante la composizione dell'Italia liberata dai Goti. La grandezza di Omero, eletto a paradigma specialmente in qualità di autore dell' 'liade ${ }^{7}$, consiste nell'aver composto un poema basato su un'azione unica - la guerra di Troia - perfetta e grande, dotata di un principio, di un mezzo e di una fine ben definiti; nell'aver operato, nei confronti di una simile materia, una

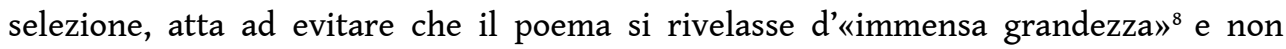
abbracciabile con uno sguardo dall'inizio alla fine - selezione che, com'è noto, riguarda gli eventi accaduti nell'arco di cinquantuno giorni, durante l'ultimo anno di guerra. Ancora, Omero è grande per aver ridotto al minimo i suoi interventi diretti come si conviene all'epica, genere per il quale, scrive Trissino glossando Aristotele, è preferibile lasciar parlare i propri personaggi piuttosto che palesarsi continuamente in prima persona. Infine, egli eccelle grazie all'uso sapiente delle similitudini e alla bellezza delle sue «menzogne»", magari impossibili ma verosimili, di certo da preferire a quelle ingannatrici, possibili ma non verosimili.

4 Quelle che risultano dalla Sesta Divisione, dunque, sono rigide regole di definizione teorica del poema eroico, derivate dallo Stagirita, alle quali Trissino, nella prassi della composizione, dimostra una radicale adesione. L'Italia liberata è il primo poema epico in Italia basato su un'unica azione con principio, mezzo e fine. Come l'Iliade, anche l'opera trissiniana racconta di una guerra, anzi della "particella» ${ }^{10}$ di una guerra, quella grecogotica (535-553), materia poi adornata di una serie di episodi satellite. La figura dell'autore all'interno del poema risulta pressoché assente, non fosse che per una serie di invocazioni alle Muse ${ }^{11}$ alle quali il poeta continuamente si appella, come già Omero, affinché lo guidino fino alla meta nel corso del suo «faticoso viaggio» ${ }^{12}$. Ancora, la similitudine ${ }^{13}$ è in assoluto la figura retorica più frequente all'interno dell'Italia liberata; i libri che contengono il resoconto delle battaglie e degli assedi si rivelano un vero e proprio tripudio di comparazioni, una delle cui principali funzioni è l'enargia, ovvero spiega Trissino in quell'ulteriore preziosa dichiarazione di poetica che è la lettera dedicatoria a Carlo $\mathrm{V}^{14}-\mathrm{l}^{\prime}$ «efficace rappresentazione» ${ }^{15}$. In altre parole, la similitudine è la figura che meglio di ogni altra permette di descrivere azioni, cose, persone con dovizia di particolari, tali da sembrare reali e da far credere al lettore di trovarvisi davanti. Non è un caso quindi la predilezione del Vicentino per quello che in fondo è uno strumento atto ad imitare la realtà secondo verosimiglianza, fine quest'ultimo della Poesia nonché principio fondante dell'intera poetica aristotelica e trissiniana.

5 Il filoellenismo e il culto iliadico di Trissino, tuttavia, non si arrestano alla pedissequa trasposizione pratica del paradigma definito dalla Poetica, di cui Omero è exemplum eccelso, ma coinvolgono L'Italia liberata dai Goti sotto ogni aspetto. Un puntuale confronto tra l'Iliade e il poema trissiniano mostra come «adorare le pedate ${ }^{16}$ di Omero per il Vicentino significhi emulare la struttura dell'opera del suo predecessore, 
riprenderne i filoni tematici principali, imitare l'architettura di singoli episodi, riprodurne lingua e stile, tanto che non esiste quasi libro dell'Iliade da cui Trissino non abbia ricavato del materiale per riscriverlo - spesso tradurlo - e inserirlo nel suo poema.

6 Anche un conoscitore di Omero non esperto ad una prima lettura dell'Italia liberata è in grado di individuare nel testo una serie di trasparenti motivi omerici: la materia bellica (e storica) che si articola in battaglie, assedi, cataloghi di eserciti, duelli in teoria risolutori, nella pratica inconcludenti; il motivo della lite tra Corsamonte ed Acquilino per l'amore di Elpidia, sulla falsariga di quella tra Achille ed Agamennone per Briseide; la conseguente rinuncia di Corsamonte a combattere e l'ambasceria a lui inviata nel vano tentativo di convincerlo a tornare; il rapimento di Elpidia; la sortita notturna di Lucillo e Tibullo in campo nemico; e naturalmente il sistema degli angeli, che per volontà divina intervengono di continuo nelle vicende umane al pari degli dei dell'olimpo.

7 L'imitatio trissiniana tuttavia non si esplica unicamente nella ripresa di topoi tematici, ma riguarda le azioni stesse dei personaggi e le parole che il poeta fa loro pronunciare. Un complessivo bilancio del debito omerico all'interno dell'Italia liberata mostra come dei ventisette libri del poema trissiniano - ma forse l'idea iniziale dell'autore era di strutturarlo in ventiquattro, come l'Iliade ${ }^{17}$ - siano quelli che trattano di guerra ${ }^{18}$ a risentire più fortemente dell'influsso di Omero, al punto da suscitare l'impressione che di originale contengano ben poco. Le battaglie tra Romani e Goti descritte da Trissino si rivelano spesso vere e proprie trasposizioni in volgare degli esametri omerici. Ogni guerriero dell'Italia liberata ha un proprio alter ego iliadico di cui ripropone fedelmente le azioni nel corso della guerra: uccide i nemici con le stesse armi e nello stesso modo del suo antenato, invoca la protezione divina, esorta i compagni, incalza i nemici, resiste o fugge, sopravvive o viene ucciso nello stesso momento in cui il suo corrispettivo dell'Iliade risulta protagonista di simili gesta. Anche nell'architettura dei duelli Trissino ricalca pedissequamente il copione omerico: le tre grandi «monomachie ${ }^{19}$ dell'Italia liberata - Acquilino contro Turrismondo (libro XVII), Achille contro Argalto (libro XX), Corsamonte contro Turrismondo (libro XXI) - ripropongono al lettore i celebri duelli di Aiace contro Ettore, di Menelao contro Alessandro, e di Achille contro Ettore. Un esempio aiuterà a chiarire più a fondo le caratteristiche dell'aemulatio trissiniana.

8 Il ventesimo libro dell'Italia liberata dai Goti, che narra il duello tra il romano Achille e il goto Argalto, nonché la lotta confusa che segue tale singolar tenzone, rappresenta una caso eclatante in questo senso, perché costruito tramite un sapiente incastro di episodi derivati da cinque diversi libri dell'Iliade: il III e il IV, il XX, il XXI e il XXII.

9 La prima parte del canto trissiniano (pp. 207-209), occupata dalla descrizione del duello vero e proprio, si limita a riecheggiare il combattimento tra Menelao e Paride del libro III dell'Iliade. Non mancano elementi comuni, come ciò che i due eserciti si giocano tramite lo scontro: nell'Iliade il marito e l'amante di Elena stabiliscono che il vincitore della tenzone potrà portarsi a casa la donna, ponendo così fine alla guerra decennale. Allo stesso modo nell'Italia liberata la sfida, lanciata da Argalto e accolta da Achille, ha lo scopo di recuperare, in caso di vittoria del guerriero goto, la città di Porto, che i Romani erano riusciti ad occupare nel corso del libro XIX. D'altro canto, in caso il vincitore risulti Achille, i Goti saranno costretti a restituire Elpidia, da loro rapita e trascinata da Taranto verso Roma. Parimenti la consacrazione dei patti tra gli eserciti, l'accurata 
misura delle dimensioni del campo di battaglia, l'invocazione a Dio da parte delle opposte schiere affinché protegga e conduca alla vittoria il proprio rappresentante sono tutti elementi che Trissino mutua da Omero. Si tratta però di rituali bellici generici, che nell'Iliade ricorrono con una frequenza altissima, non solo nel libro III, e che lo stesso Trissino recupera, nel medesimo ordine, ogni qual volta si accinga a descrivere un duello ${ }^{20}$. In effetti poi i due scontri si svolgono e si concludono in maniera differente: con la morte di Argalto quello trissiniano, con l'intervento salvifico di Afrodite a favore di Paride il secondo.

Ciò che induce a pensare che l'archetipo della contesa tra Achille e Argalto sia lo scontro Menelao-Paride, piuttosto che un altro degli innumerevoli duelli iliadici, sono gli eventi successivi alla battaglia. La seconda macrosequenza (pp. 209-210) del ventesimo libro dell'Italia liberata, infatti, è da considerarsi un vero adattamento in endecasillabi del testo omerico. Si consideri il libro IV dell'Iliade: alla fine del duello interviene Atena, persuadendo il troiano Pandaro a violare i patti precedentemente giurati e a scagliare una freccia contro Menelao, non senza promettere ad Apollo di

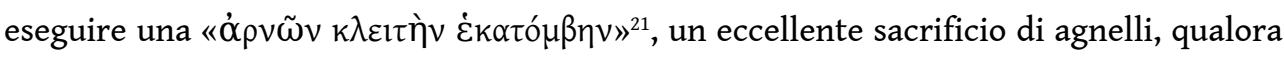
un giorno riesca a tornare in patria. In Trissino è l'angelo Nemesio a rivestire il ruolo della Pallade e a convincere l'arciere Ablavio a dare la morte ad Achille con suo dardo, per vendicare la triste fine di Argalto, rimasto ucciso nel corso della tenzone. Il goto non esita ad obbedire e il sacrificio che anch'egli, come il suo corrispettivo omerico, promette a Dio è inserito in un contesto geografico nuovo e ben conosciuto dal Trissino:

Poi pregò il Re del ciel con tai parole.

«Eterno Re, ch'a l'opre de' mortali

Dai sempre, quando vuoi, felice effetto,

Drizza la mia saetta entr' a la carne

Del fiero Achille, e fa, ch'io gli dia morte,

Per far vendetta del feroce Argalto,

Ch'era di sangue a me tanto congiunto;

Che com'io torni in Padoa, faccio voto,

Di farti fare un sacrificio grande

Dentr'a Santa Sofia vicin' al fiume;» [... ${ }^{22}$.

Le conseguenze della violazione dei patti sono identiche in Trissino e in Omero: l'Achille trissiniano, come Menelao, viene ferito solo superficialmente dalla «sætta amara ${ }^{23}$, grazie ad un intervento divino. Corsamonte, come Agamennone, si dispera credendo il compagno morto. Dopo l'assicurazione di Achille-Menelao a CorsamonteAgamennone di non aver ricevuto lesione mortale, i Romani-Achei si affrettano a chiamare un medico (Teodetto-Macaone) che curi il ferito.

11 L'ultima parte del libro trissiniano (pp. 210-216) si concentra sulle gesta di Corsamonte. A seguito del duello esplode una feroce battaglia tra Romani e Goti, e il duca di Scizia combatte in prima linea desideroso di vendicare l'affronto subito da Achille. Trissino costruisce l'episodio riscrivendo e letteralmente incastrando tra loro, come in una sorta di mosaico testuale, gruppi di versi omerici tratti dai libri XX, XXI e XXII dell'Iliade. Si ricordi che l'alter ego iliadico di Corsamonte è l'Achille omerico: non è un caso quindi che le eroiche imprese di Corsamonte del libro XX riproducano quelle compiute dal Pelìde al suo rientro in guerra, quando, morto Patroclo, si verifica la cosiddetta «interruzione dell'ira».

In un primo momento il duca di Scizia è impegnato contro Totila, così come Achille nel ventesimo libro dell'Iliade si scontra con Enea. Dopo un acerbo scambio di battute tra le due coppie di guerrieri ha inizio la lotta. Totila ed Enea sono in evidente svantaggio, 
tuttavia, riescono a mettersi in salvo grazie ad un tempestivo intervento divino: l'angelo Adrastio e Poseidone, infatti, versano sugli occhi del nemico una «nebbia

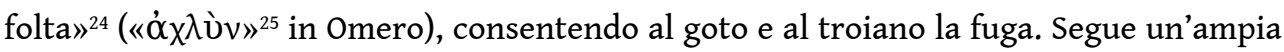
sequenza dedicata alle stragi di Corsamonte e Achille: i due eroi, sbigottiti di fronte all'improvvisa scomparsa di Totila ed Enea, si dedicano al massacro del resto dell'esercito nemico. Così, Fabalto e Ifitione muoiono per una ferita alla testa:

E lo ferì con l'asta ne la testa,

E tutta la passò di banda in banda; [...].

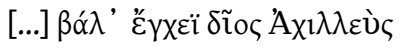

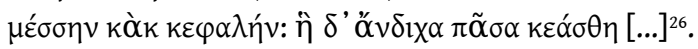

Sabinaco e Demoleonte per un colpo alla tempia:

[...] con l'asta lo punse entr'a una tempia,

E ruppe l'osso prima, e poi la tinse

De le cervelle sua, ch'eran quiv'entro [...].

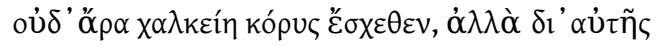

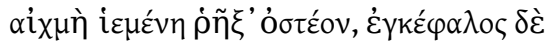

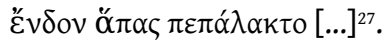

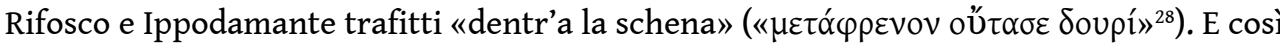
via, fino ad arrivare a Materno e Troo, i quali entrambi chiedono pietà, ma vanno incontro a diversa sorte: Corsamonte infatti si lascia commuovere da chi gli abbraccia le ginocchia, Achille invece lo priva della vita ${ }^{29}$.

Se la breve sequenza del combattimento tra Corsamonte e Vitige (p.214) è tutta trissiniana, le corrispondenze con l'Iliade - non più con il libro XX, ma con il XXI riemergono verso la fine dell'episodio, a partire dalla similitudine di Corsamonte massacratore di Goti con il fuoco che si sparge in una foresta (p. 215). Mentre il goto Unigasto ordina che vengano aperte le porte del vallo, affinché il suo popolo possa correre al riparo delle mura - e Priamo fa lo stesso con le porte Scee - Corsamonte si scaglia contro Bisandro - in realtà l'angelo Latonio sotto mentite spoglie - e Achille contro Agenore-Apollo. Una volta tratti in salvo Goti e Troiani, le due divinità rivelano al proprio oppositore la loro reale natura: le "parole acerbe» con cui Latonio si rivolge al duca di Scizia, ammonendolo a non combattere contro un «messo di Dio», e con le quali si chiude il canto, riproducono quelle che Apollo pronuncia all'inizio del libro ventiduesimo dell'Iliade:

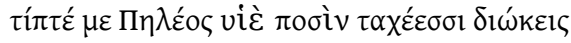

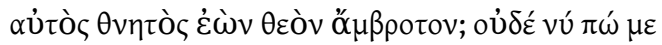

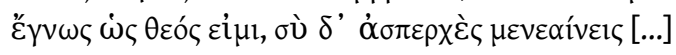

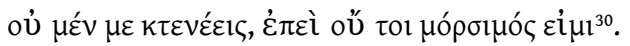

Il confronto proposto, emblematico di come Trissino vada ad adattare al suo poema materia narrativa omerica, è solo uno dei tanti casi rintracciabili all'interno dell'Italia liberata. Si pensi ad esempio alla figura di Anticalo, tutta ricalcata sul Tersite omerico: al pari di questo brutto e sempre pronto a rendersi odioso, nel libro XIV dell'Italia egli si merita una dura e pubblica lezione da parte di Traiano, così come Tersite la riceva da Odisseo (Il. II, 211-277). All'interno del medesimo libro, l'ambasceria di Ciro e Traiano presso Corsamonte, con l'obiettivo di convincerlo a rientrare al campo, è la copia perfetta di quella di Odisseo, Aiace e Fenice presso Achille (Il. IX, 182-713). Ancora, il Vicentino riproduce per intero il canto $\mathrm{X}$ dell'Iliade, relativo alla celebre sortita notturna di Odisseo e Diomede. In questo caso l'imitazione del modello risulta scorporata in due diversi libri dell'Italia: il XIX, in cui l'impresa di Mundello e Traiano ricalca la prima metà dell'episodio omerico, e il XIII, con protagonisti Lucillo e Tibullo, 
che guarda invece alla parte finale del canto X dell'Iliade. Si citi infine il libro XXIII dell'opera trissiniana, in cui il poeta narra lo svolgersi dei giochi funebri in onore di Corsamonte: esso è interamente costruito sul canto XXIII dell'Iliade, relativo ai giochi che Achille fa celebrare in memoria del'ucciso Patroclo.

Il confronto sopra proposto rende inoltre obbligatoria una riflessione sulla qualità dell'imitatio omerica trissiniana. Molto duri, in tal senso, i giudizi già formulati da Zatti e da Gigante, i quali parlano rispettivamente di «classicismo all'insegna della "affettazione" $»^{31}$, e di materiali della tradizione «utilizzati in modo grezzo con pochi, indispensabili aggiustamenti [...] che quasi sempre appaiono male assemblati, se non addirittura ridicoli $»^{32}$. Al di là dei risvolti quasi umoristici che talvolta l' «"ansia" trissiniana di emulazione dell'archetipo ${ }^{33}$ produce come conseguenza - e su cui già gli antichi commentatori non mancarono di puntare il dito ${ }^{34}$ - quanto emerge dalla nostra analisi non si discosta dalle valutazioni offerte dai due studiosi. L'aderenza trissiniana alla fonte omerica si traduce troppo spesso in incapacità di rielaborazione del modello. Gli episodi iliadici non risultano ben amalgamati all'interno del tessuto narrativo dell'Italia; anzi, si può dire che non lo siano affatto, risultando immediatamente riconoscibili anche a chi, di Omero, non è esperto lettore. Il poeta preleva passi dell'rliade, li adatta all'italiano e all'endecasillabo, rimaneggiandoli quel tanto che serve a renderli conformi alla materia narrativa del suo poema. Quindi li ricuce meccanicamente in questo o in quel libro dell'Italia, come in una sorta di patchwork narrativo. In questo, per usare di nuovo le parole di Gigante, consiste la debolezza del poeta vicentino: nell'aver sperimentato «un confronto impossibile con l'Iliade», nel suo tentativo di «rifar parlare Omero con la propria voce, lasciando che il fantasma del Modello lo schiacciasse, trionfante, con tutta la sua forza» ${ }^{35}$.

\section{Lo Zibaldone autografo trissiniano come laboratorio di traduzione omerica}

16 Allo studio del rapporto dell'Italia liberata con la fonte omerica hanno contribuito, come si accennava all'inizio della trattazione, i manoscritti trissiniani conservati presso la Biblioteca Nazionale Braidense di Milano, noti come Fondi Castiglioni 8/1, 8/2 e 8/3. Si tratta di codici contenenti lettere, testi in prosa e in versi di vario genere, appunti, schemi e sperimentazioni versificatorie di mano del Vicentino. Interamente autografo, in particolare, è il Castiglioni $8 / 1^{36}$, noto altrimenti come Zibaldone autografo dell'Italia liberata, alla cui trascrizione e commento sto dedicando un importante capitolo della mia tesi dottorale. Gli altri due volumi raccolgono carte autografe (soprattutto lettere private o ufficiali e testi letterari) insieme a fogli vergati da altre mani: tra queste, fanno notare Maria Lieber e Christian Weyers - due dei pochi studiosi ad essersi occupati dei manoscritti ${ }^{37}$-sono riconoscibili quelle di Francesco Testa e di Bartolomeo Zigiotti, eruditi particolarmente attivi nel riordino del materiale autografo trissiniano. Ovvi motivi di spazio mi impediscono di tracciare la storia dettagliata dei tre manoscritti ${ }^{38}$, così come di addentrarmi in un'esauriente descrizione esterna ed interna dei codici. Mi limito perciò ad accennare alla struttura e al contenuto del primo dei tre, quello Zibaldone dell'Italia liberata il cui titolo è sufficiente a far intuire la sua preziosità ai fini della mia ricerca.

17 «Zibaldone » significa mescolanza, miscuglio, disordine. Una simile etichetta sembra adattarsi perfettamente al primo dei tre volumi trissiniani: le sue 189 carte infatti - 
organizzate alfabeticamente come un rubrica, delle quali però meno della metà risulta vergata $^{39}$ - sono disposte, almeno in apparenza, secondo un ordine casuale e possono risultare inizialmente di difficile decifrazione. In realtà c'è un fil rouge che unisce la quasi totalità dei fogli del Castiglioni 8/1: il codice assembla appunti autografi in vista della stesura dell'Italia liberata. Esso rappresenta un vero e proprio cantiere di progettazione del poema, in cui Trissino raccoglie annotazioni di vario genere: indici e schemi relativi alla struttura dei diversi libri del poema; annotazioni circa lo spazio in cui ambientare le vicende (l'impero d'Oriente; la Roma del VI secolo); cronologia degli eventi narrati; elenchi dei personaggi dell'opera e delle rispettive regioni geografiche di provenienza; finanche schemi e disegni della disposizione degli eserciti durante le battaglie; e sopratutto studi e prove di versi da inserire nel poema, l'elemento più prezioso in assoluto perché, qualora messi a confronto con la loro versione definitiva all'interno dell'opera, fanno luce sul modus poetandi dell'autore.

Lo studio dello Zibaldone si è rivelato di notevole interesse sotto vari punti di vista: in primo luogo il codice consente un ingresso privilegiato nell'officina scrittoria trissiniana. Secondariamente - e in maniera inaspettata - il manoscritto si è dimostrato uno strumento di inestimabile valore per l'indagine delle letture trissiniane e l'approfondimento di un argomento rispetto al quale «nemmeno in tempi lontani sono state avviate ricerche significative, al punto che oggi appare una sorta di terra incognita» ${ }^{40}$ : la biblioteca del Trissino. In molte occasioni, infatti, le carte dello Zibaldone lasciano trapelare delle fonti che aiutano lo studioso nella ricostruzione dei gusti librari trissiniani, permettendo di intuire quali autori e quali volumi, se non appartenevano alla biblioteca del Vicentino, per lo meno gli passavano per le mani.

Legato a quest'aspetto, nonché più specificamente pertinente alla materia del presente articolo, è il terzo motivo d'importanza del Castiglioni 8/1. Molti dei suoi abbozzi si rivelano di estremo interesse anche per la ricchezza di informazioni che sono in grado di fornire circa le caratteristiche dell'imitatio omerica all'interno dell' Italia liberata. Molte carte del codice, infatti, si rivelano ancora più preziose qualora si esegua un confronto incrociato tra il testo manoscritto in esse contenuto, $i$ versi corrispettivi dell'Italia liberata così come compaiono nella versione definitiva dell'opera, e il testo dell'Iliade. Alla luce di un simile raffronto gli appunti trissiniani dello Zibaldone risultano essere non solo prove di versi in vista della stesura del poema, ma veri e propri esempi di riscrittura - talvolta di traduzione - del testo omerico, che Trissino prova e riprova ad adattare agli endecasillabi e alla materia della sua futura opera. Esempi di riscrittura che incarnano spesso uno stadio intermedio dell'Italia liberata, più aderente ancora al poema di Omero di quanto già non lo sia la versione ultima dell'epopea trissiniana.

20 A suggerire che il confronto con l'Iliade costituisca la chiave di lettura adeguata per quelli che, diversamente, potrebbero sembrare gruppi di versi sparsi è lo stesso Trissino. Tra le carte più celebri del codice spiccano infatti la 96v e la 180r. La prima contiene un elenco di nomi di personaggi dell'Italia liberata, Romani e Goti, a ciascuno dei quali corrisponde un eroe omerico, acheo o troiano: l'idea di fornire ogni guerriero del poema di un alter ego iliadico era quindi nelle precise intenzioni dell'autore. La c. $180 r$, scritta in greco, è strutturata su due colonne: quella di destra riporta il primo verso, o parole del primo verso, di tutti i ventiquattro libri dell'liade; quella di sinistra brevi indicazioni, sempre in greco, circa il contenuto di ciascuno di questi libri ${ }^{41}$. Trissino, quindi, sembra assumere Omero "per Duce e per Idea» già nelle fasi di 
abbozzo e di studio di versi per l'Italia liberata. Qualche esempio aiuterà a capire in che misura.

21 Si prenda in considerazione la carta $14 r$. Essa, contenente prove di similitudini, è aperta da un appunto autografo, quasi un ammonimento che Trissino fa a sé stesso. Citando la

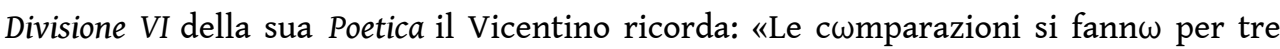

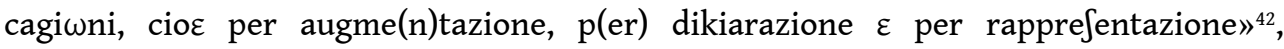
promemoria emblematico del solido legame che per quest'autore unisce teoria e prassi poetica. Segue un elenco di tali «cwmparazioni», tutte inserite pressoché identiche all'interno dell'Italia liberata, con un'unica eccezione:

Si come quand $\omega$ fulmina il marit $\omega$

De la bella Giunone, wnde difcende

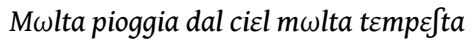

0 quand $\omega$ i vapwr freddi in spesse falde

Fioccan di neve $\varepsilon$ fan la terra bianca

$\mathrm{C} \omega$ sì spessi i s $\omega \int$ piri ufcian del pett $\omega$

Del capitani $\omega, \varepsilon$ lj $\omega$ ndeggiava il cuwre

$\varepsilon$ s'admirava riguardand $\omega$ al camp $\omega$

$\varepsilon$ ved $\varepsilon$ nd $\omega$ quel fuoc $\omega \varepsilon^{\prime}$ l gran rum $\omega$ re

che facean quivi d'huomini $\varepsilon$ di trwmbe.

Ma quando rifguardava poi la terra

$\varepsilon$ vedea le sue genti Jbigwtite

Molti capelli si trahea di t $\varepsilon f t a$.

Gemendo f $\omega r$ te $\varepsilon$ rimira(n)do al ciel $\omega$

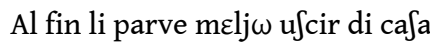

$\varepsilon$ ritrwvare il buon conte d'Ifaura

Per sec $\omega$ inve tigar qualche $c \omega(n) \operatorname{silj\omega }$

Che dia salute a q(ue)lla afflitta gente.

E come quando fulmina il marito

De la bella Giunone, onde discende

Molta pioggia dal ciel, molta tempesta;

0 quando $i$ vapor freddi in spesse falde

Fioccan di neve, e fan la terra bianca,

Così frequenti ognor saette, e lance

Pioveano intorno al capitanio eccelso.

Ma Dio non si scordò del tuo periglio,

Belisario gentil, né quello eterno

Angel Palladio, anzi ei ti stava a canto,

$\mathrm{E}$ facea gir molte saette al vento,

E molte lance rivoltava, e molte

facea lente arrivar dentr'al tuo scudo.

Né la tua bella compagnia del sole

Fu pigra ad ajutarti, anzi ogniun d'essi

Poneanti i scudi, e le persone avanti,

E riceveano in se molte percosse,

Che venute sarian contra il tuo petto[... $]^{43}$.

Il confronto mostra come, nel passaggio dall'abbozzo alla versione definitiva della similitudine, il secondo elemento di paragone cambi. Nel Castiglioni 8/1 l'abbondanza di pioggia e di neve durante una tempesta viene assimilata alla frequenza dei gemiti di un Belisario («il capitanio») disperato, che guarda con preoccupazione il suo esercito «sbigotito». Nell'Italia liberata, invece, ad essere «spessi» come i fiocchi di neve che imbiancano il terreno sono i dardi e le saette che i Goti scagliano contro il generale nemico nel corso dell'assedio del libro dodicesimo (p. 123). La netta differenza tra i due 
gruppi di versi assume un significato nuovo qualora si metta a confronto la similitudine dello Zibaldone con quella che apre il libro X dell'Iliade:

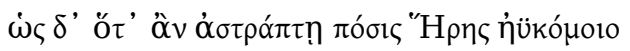

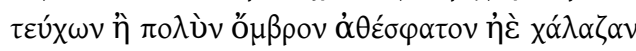

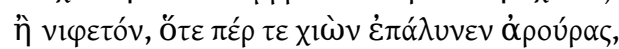

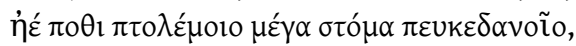

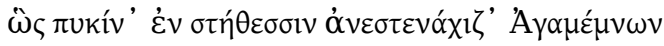

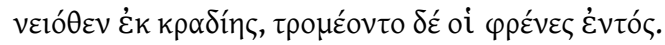

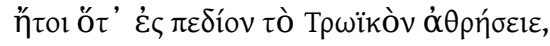

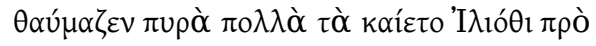

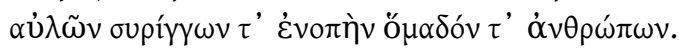

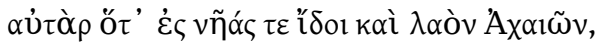

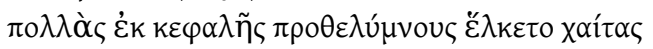

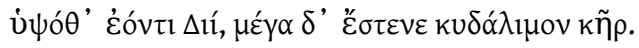

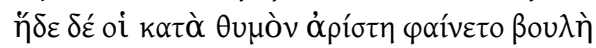

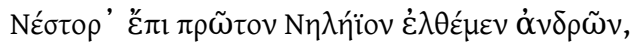

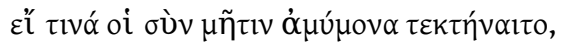

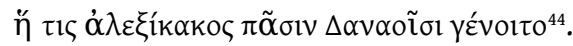

Omero ritrae l'inquietudine di Agamennone: il capo degli Achei, turbato a seguito del

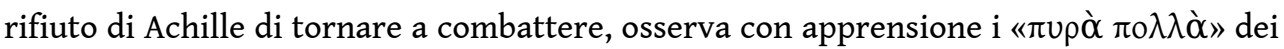
Troiani che ardono illuminando la pianura. Decide infine di chiedere consiglio al saggio Nestore, unico in grado di alleviare le sue preoccupazioni circa la riuscita dell'impresa achea.

Accostando il testo omerico alla similitudine dello Zibaldone trissiniano risulta evidente l'operazione compiuta dal Vicentino: Trissino traduce Omero quasi alla lettera, adattando i sentimenti dell'Atride a quelli del suo Belisario. Il cuore del «capitanio

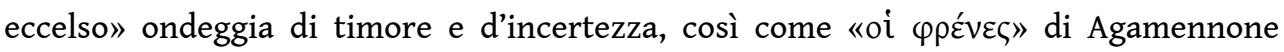

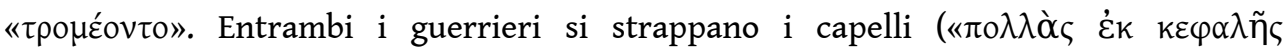

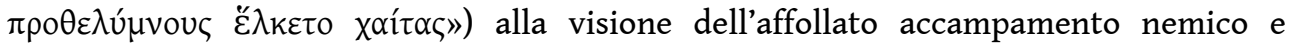

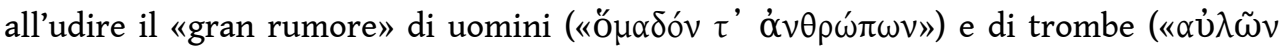

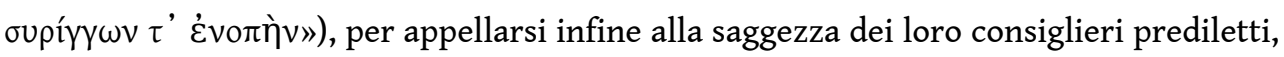
Nestore e Paulo, conte d'Isaura.

La similitudine del Castiglioni 8/1, quindi, risulta ben più fedele a quella dell'Iliade che non alla versione licenziata poi nell'Italia liberata. Le ragioni che hanno spinto Trissino a modificare il secondo elemento di paragone, più che rispondere ad esigenze testuali, sono da individuare probabilmente nell'immagine di Belisario che emerge dalla prima comparazione. Si ricordi infatti che nell'Italia liberata il capo dei Romani è l'incarnazione dell'ordine, dell'equilibrio, della razionalità, il paradigma perfetto del buon comandante: mantiene i suoi uomini sani, forti, pronti alla battaglia, sa ascoltare e farsi ascoltare, non teme i pericoli ${ }^{45}$. È evidente che il ritratto di un Belisario piangente e scomposto, che sulla falsariga dell'Agamennone omerico si strappa i capelli di fronte al suo popolo, poco si confà al modello di decoro sopra descritto: da qui la decisione di Trissino di eliminare del tutto questi versi.

Un ulteriore prezioso esempio di riscrittura omerica compare nella carta $37^{1} v$, foglio autografo in origine non appartenente al manoscritto, ma aggiunto più tardi. Il recto e il verso della pagina presentano un elenco di brevissime similitudini o prove di versi, di uno o due endecasillabi ciascuna, inserite poi in vari libri dell'Italia liberata senza un apparente ordine. Per chiarezza si riproduce per intero il verso del foglio in questione: 
Le navi poi c $\omega n$ le g $\omega$ nfiate vele

Givan per l'ampia schena di Nettuno

$\varepsilon$ sempre difiava alcun $\mathrm{d} \varepsilon$ i Gotthi

$C \omega$ prir di giacci $\omega \varepsilon$ di perpetua notte.

Il mar che $c \omega l \omega r a t \omega \varepsilon$ di [ $>\varepsilon$ del c $\omega l \omega r$ de le] viole

Ma chi fu il prim $\omega$ che mandasse a m $\omega$ rte

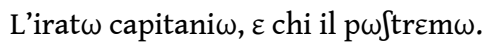

$\varepsilon$ ntr $\omega$ ne la battalja c $\omega$ me un vent $\omega$

Ch'entri nel mare $\varepsilon$ che c $\omega m m \omega v a$ l' $\omega$ nde

$\mathrm{C} \omega$ sì il sign $\omega \mathrm{r} \mathrm{c} \omega \mathrm{n}$ l'alte sue parole

Sveljò in ciafcun $\omega \varepsilon$ l'anim $\omega \varepsilon$ le forze

$\varepsilon$ c $\omega m e$ il cacciat $\omega r$ che exhorta i cani

$\mathrm{C} \omega$ ntra i cingiali asperimi $\varepsilon$ i lewni

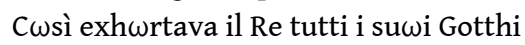

C $\omega$ ntra l'ardite f $\omega r z e$ de i R $\omega$ mani

$\mathrm{S} \varepsilon(\mathrm{m})$ pre mandand $\omega$ l'ultim $\omega$ a la m $\omega$ rte

M $\omega$ lti cadean $\omega$ supini [> giù] de i l $\omega$ r cavalli

Per le perc $\omega$ sse del feroce Achille.

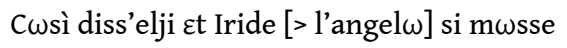

Per nwn difubidire ai suoi precetti.

L'hafta nutrita al vent $\omega$ haveva ${ }^{a}$ in man $\omega$

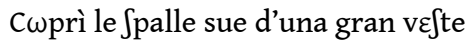

Di velut $\omega$ r $\omega$ at $\omega$ ad $\omega$ rna d'or $\omega$

Era g $\omega$ vernat $\omega$ r di quelle genti

Reverito dal ${ }^{1 \omega r}$ p $\omega$ p $\omega l$ c c $\omega m e^{\text {su } \omega}$ un Di $\omega$

In realtà un ordine c'è e diventa trasparente qualora si assuma ancora una volta l''liade come guida di lettura. I versi trissiniani si rivelano infatti veri e propri esercizi di traduzione o riscrittura di esametri omerici, tutti tratti dal libro XI dell'Iliade ${ }^{46}$. Emblematico in tal senso il distico:

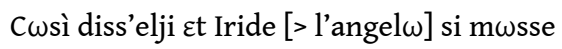

Per nwn difubidire ai suoi precetti.

Dalla coppia di endecasillabi emerge con evidenza la fonte omerica: essi riproducono il

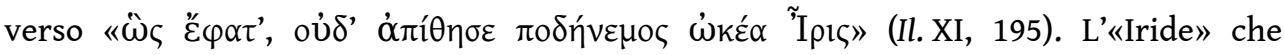
compare nell'endecasillabo trissiniano altro non è che la versione volgarizzata dell' 'ٓ $1 \rho ı$ omerica, dea dell'arcobaleno e messaggera degli dei, che Zeus spesso invia sulla terra perché riferisca ai mortali la sua volontà. Proprio il nome della dea diventa spia tangibile del fatto che Trissino stia traducendo Omero. Il Vicentino semplifica gli epiteti

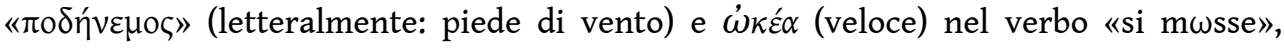
mentre «non disubidire» equivale al greco «oủ $\delta$ ' $\dot{\alpha} \pi i ́ \theta$ $\sigma \varepsilon »$. Quanto a «l'angel $\omega »$, che Trissino propone in alternativa a «Iride», è indizio di come l'autore intenda adattare la fonte omerica alle proprie esigenze: nell'Italia liberata a volare sulla terra per ubbidire ai comandi divini dovrebbe essere uno dei tanti angeli che affollano le scene di guerra.

In realtà il distico in questione è uno dei pochi ${ }^{47}$ della carta $37^{1} v$ a non essere poi inserito, nemmeno in forma modificata, nella versione del poema data alle stampe. Simile il caso del verso:

Il mar che $c \omega l \omega r a t \omega \varepsilon$ di [ $\varepsilon$ del c $\omega l \omega r$ de le] viole

Anch'esso non trova spazio nell'edizione definitiva del poema trissiniano. Una ricerca testuale all'interno dell'Italia liberata ha mostrato come l'unica occasione in cui il mare viene descritto nei termini di livido, scuro è nel libro XV, quando l'autore si sofferma sullo stemma che orna lo scudo di Totila: «[...] Et avea in mezo la caribde orrenda,/ Di 
color perso, co i feroci scogli $»^{48}$. Difficile però interpretare il sintagma «di color perso» quale versione ultima di «del c $\omega l \omega r$ de le viole», seppur la reminescenza omerica è indubbia ${ }^{49}$. L'aspetto più interessante dell'abbozzo dello Zibaldone, infatti, al di là della sua assenza nell'Italia liberata, è l'evidente marchio omerico che contiene: Trissino tenta

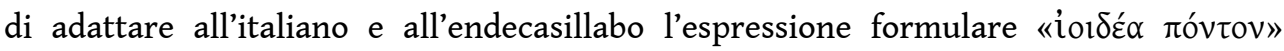

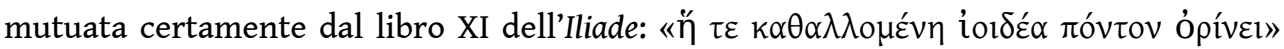
$\left(\right.$ Il. XI, 298) ${ }^{50}$.

30 Altro caso di volgarizzamento di una formula omerica è il verso:

L'hafta nutrita al vent $\omega$ haveva ${ }^{a}$ in man $\omega$

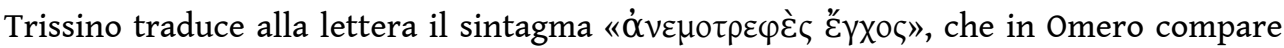
con una certa frequenza ${ }^{51}$, ma che, quasi senza incertezza, si può ipotizzare sia

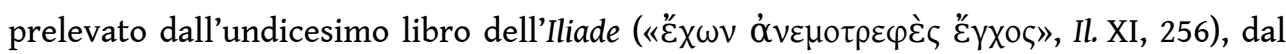
momento che tutte le altre prove di versi della carta $37^{1} v$ mostrano corrispondenze con tale canto omerico. Peraltro, a differenza dei due esempi precedenti, questo verso dello Zibaldone compare anche nell'Italia liberata e Trissino, sulla scia di Omero, ne fa un uso formulare ${ }^{52}$. Esso infatti ricorre all'inizio della «orribil zuffa» tra Romani e Goti che occupa per intero il libro XII, quando il goto Totila si scaglia contro il nemico Filodemo: «E così fieramente lo percosse/ Con la dura asta sua nutrita al vento/ Che gli fu forza

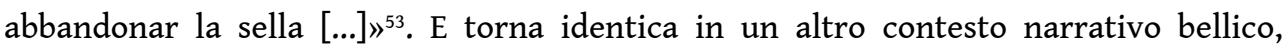
ovvero durante la battaglia del libro XV. L'arma in questo caso appartiene al valoroso Belisario che fa letteralmente strage di nemici intorno a sé: «Uccise ancor Cassandro, e Tamberlano/ E Girotto, e Grumalto, e Bellapecca,/ Tutti con l'asta sua nutrita al vento» ${ }^{54}$.

31 Il fatto che tutte le prove di versi della carta $37^{1} v$ derivino dal libro XI dell'Iliade rende doverosa una riflessione circa il modo in cui Trissino lavorasse sul testo omerico. Egli era un conoscitore esperto della lingua e della letteratura greca e non c'è dubbio che conoscesse larghe porzioni dell'Iliade a memoria ${ }^{55}$. Nulla esclude, quindi, che nel corso di questo lavoro di traduzione e trasformazione della fonte procedesse basandosi sulla memoria. Tuttavia, nel caso specifico della carta in questione, la brevità dei versi trissiniani, il fatto che siano tutti ricavanti dall'undicesimo libro, e che compaiano spesso nel medesimo ordine di apparizione del testo omerico - seppur non si tratti di versi consecutivi - fanno supporre che il Vicentino stesse lavorando con il poema iliadico sotto mano, selezionando, mano a mano che vi si imbatteva, esametri funzionali alle esigenze dell'Italia liberata e provando ad adattarli all'italiano.

Terminata questa panoramica di esempi che - lungi dal voler essere esaustiva - riesce però a rendere l'idea di quella che all'inizio del capitolo si era definita devozione trissiniana nei confronti di Omero, si possono trarre alcune conclusioni. Le carte autografe sopra analizzate sono da considerarsi alla stregua di fogli di lavoro in vista della stesura del poema. Trissino preleva esametri dell'Iliade, li traduce talvolta alla lettera, talvolta in maniera più libera, e li mette in endecasillabi adattando personaggi e vicende a quelli che ha in mente per l'Italia liberata. Così facendo si crea un repertorio di similitudini e di versi da cui attingere nella fase di effettiva redazione dell'opera. Si riconsideri infatti per un momento la c. $37^{1} \mathrm{v}$ : la serie di brevi versi - in apparenza del tutto decontestualizzati - che essa propone, grazie al confronto con l'Iliade si rivela essere un vero e proprio laboratorio di traduzione. Il fatto, inoltre, che nove volte su dodici i versi dello Zibaldone ricompaiano nel poema, avvalla l'ipotesi che Trissino si stesse creando una sorta di magazzino di versi ed espressioni formulari tratte da Omero, con l'intento di attingervi all'occorrenza durante la fase di scrittura del poema. 
E - come si è visto nel caso della similitudine di c. 14r - l'aspetto ancora più interessante della questione è che i fogli del Castiglioni 8/1 spesso riflettono una fase intermedia del lavoro trissiniano, a metà strada tra Omero e la versione finale dell'opera. Entrare nell'officina dell'autore, dunque, significa questo: poter contare su uno strumento in grado di mettere in luce eventuali dubbi e incertezze dell'autore nella prima fase di traduzione omerica; evidenziare come muta l'aderenza di Trissino all'Iliade nel passaggio dall'abbozzo ai versi nella loro forma definitiva; mostrare che l'imitatio omerica del Vicentino non riguarda solo la costruzione di alcuni episodi ma la stessa progettazione di singoli gruppi di versi, finanche la lingua del suo poema.

\section{BIBLIOGRAPHIE}

ARISTOTELE, Poetica, a cura di D. LANZA, Milano, Rizzoli, 1987.

BALDASSARRI, G., Il sonno di Zeus. Sperimentazione narrativa del poema rinascimentale e tradizione omerica, Roma, Bulzoni, 1982.

GIRALDI CINZIO, G., Discorso intorno al comporre de' romanzi, delle commedie, e delle tragedie, e di altre maniere di poesia, in ID., Scritti critici, a cura di C. GUERRIERI CROCETTI, Milano, Marzorati, 1973. CORRIERI, A., Rivisitazioni cavalleresche ne L'Italia liberata da' Gotthi di Giangiorgio Trissino, «Schifanoia», XXXIV-XXXV, 2008, pp. 183-192.

CROCE, B., L'Italia liberata dai Goti, in ID., Poeti e scrittori del pieno e tardo Rinascimento, Bari, Laterza, 1945, vol. I.

DI SANTO, F., Il poema epico rinascimentale e l'iliade: da Trissino a Tasso, Tesi di Dottorato in Memoria Culturale, Università di Pisa, A.A. 2011/2012, Relatore Prof. G. PADUANO.

GIGANTE, C., Un'interpretazione dell'Italia liberata dai Goti, in ID., Esperienze di filologia cinquecentesca. Salviati, Mazzoni, Trissino, Costo, Il Bargeo, Tasso, Roma, Salerno, 2003, pp. 46-79.

LIEBER M., WEYERS C., Giovan Giorgio Trissino: i manoscritti Castiglioni 8/1, 8/2, 8/3 della Biblioteca

Braidense di Milano, in M. LIEBER, W. HIRDT (a cura di), Kunst und Kommunikation. Betrachtungenzum Medium Sprache in der Romania. Festschrift zum 60. Geburstag von Richard Baum, Tübingen, Stauffenburg, 1997, pp. 221-254.

MIGLIORINI, B., Le proposte trissiniane di riforma ortografica, in «Lingua Nostra», XI, 4, 1950, pp. 77-81. MORSOLIN, B., Giangiorgio Trissino. Monografia di un gentiluomo letterato del secolo XVI, Firenze, Le Monnier, 1894.

MUSACCHIO, E., Il poema epico ad una svolta: Trissino tra modello epico e modello virgiliano, «Italica», 80, 2003, pp. 334-352.

MUSACCHIO, E., Lo stile del nuovo poema epico rinascimentale, «Letteratura Italiana Antica», VI, 2005, pp. 369-389.

OMERo, Iliade, a cura di M. G. CIANI, commento di E. AVEzzÙ, Marsilio, Venezia, 1990. 
TOMASI, F., Gian Giorgio Trissino, in M. MOTOLESE, P. PROCACCIOLI, E. RUSSO (a cura di), Autografi dei letterati italiani. Il Cinquecento, vol. II, Roma, Salerno, 2013, pp. 369-385.

QUONDAM, A., La poesia duplicata. Imitazione e scrittura nell'esperienza del Trissino, in N. POZZA (a cura di), Atti del Convegno di Studi su Giangiorgio Trissino, Vicenza, Accademia Olimpica, 31 marzo-1 aprile 1979, Vicenza, Neri Pozza, 1980, pp. 67-109.

TRISSINO, G. G., La Italia liberata da Gotti, in S. MAFFEI (a cura di), Tutte le opere di Giovan Giorgio Trissino, gentiluomo vicentino, non più raccolte, Verona, appresso Jacopo Vallarsi, vol. I, p. 1-296. TRISSINO, G. G., Poetica (V-VI), in B. WEINBERG (a cura di), Trattati di retorica e di poetica, vol. II, Bari, Laterza, pp. 5-90.

VITALE, M., L'Omerida italico: Gian Giorgio Trissino: appunti sulla lingua dell'Italia liberata da' Gotthi, Venezia, Istituto di Lettere Scienze ed Arti, Classe di scienze morali lettere ed arti, 2010.

ZATTI, S. L'imperialismo epico del Trissino, in ID., L'ombra del Tasso. Epica e romanzo nel Cinquecento, Milano, Mondadori, 1996, pp. 56-110.

\section{ANNEXES}

\section{Corrispondenze tra Fondo Castiglioni 8/1: c. $37^{1}$ v, I'lliade (II) e la versione definitiva de l'Italia liberata (IL)}

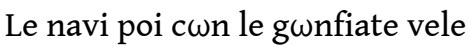

Givan per l'ampia schena di Nettuno

Il: -

IL: -

$\varepsilon$ sempre difiava alcun d $\varepsilon$ i Gotthi

$C \omega$ prir di giacci $\omega \varepsilon$ di perpetua notte.

Il: -

IL, Libro XV (p. 158)

Con ardente disio di ricoprirli

Tutti di giaccio, e di perpetua notte, [...].

$\varepsilon$ del col $\omega$ r de le

Il mar che $c \omega l \omega r a t \omega \varepsilon$ di viole

Il, XI, 297-298

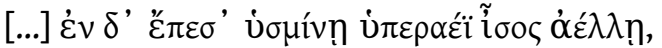

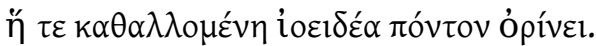

IL, Libro XV (p.158)

Et avea in mezo la caribde orrenda,

Di color perso, co i feroci scogli, [...].

Ma chi fu il prim $\omega$ che mandasse a mwrte

L'irat $\omega$ capitani $\omega, \varepsilon$ chi il p $\omega \int \operatorname{trcm} \omega$.

Il, XI, 299-300

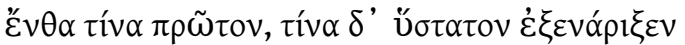

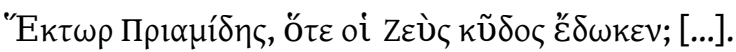




\section{IL, Libro XVIII (p.191)}

Ma chi fu, Muse, il primo, e chi il postremo, Che morti fur da Turrismondo allora?

$\varepsilon n t r \omega$ ne la battalja c $\omega m e$ un vent $\omega$ Ch'entri nel mare $\varepsilon$ che $c \omega m m \omega v a$ l' $\omega$ nde

Il, XI, 297-298

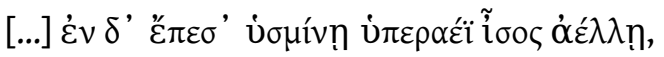

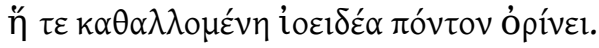

IL, Libro XVIII (p. 191)

Et urtò in essi, come fosse un vento, Ch'entri nel mare, e che commuova l'onde.

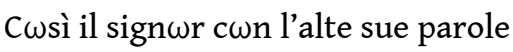
Sveljò in ciafcun $\omega \varepsilon$ l'anim $\omega \varepsilon$ le forze Il, XI, 291

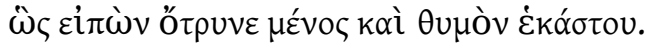
IL: -

$\varepsilon$ c $\omega$ me il cacciat $\omega r$ che exhorta i cani

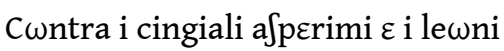

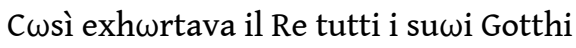
C $\omega n$ tra l'ardite f $\omega r z e$ de i R $\omega$ mani Il, XI, 292-295

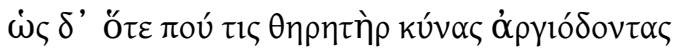

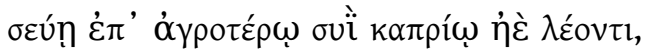

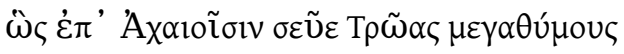

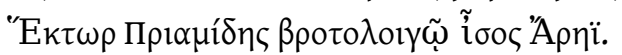
IL, Libro XVIII (p.191)

E come il cacciatore esorta i cani Contra i cengiali asperrimi, o i leoni, Così esortava il Re tutti i suoi Gotti, Contra l'ardite forze de i Romani.

$\mathrm{S} \varepsilon(\mathrm{m})$ pre mandand $\omega$ l'ultim $\omega$ a la m $\omega$ rte

Il, XI, 177-178

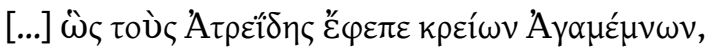

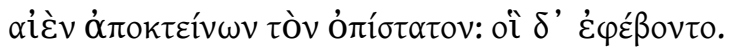

IL, Libro XV (p.158)

Sempre mandando gli ultimi a la morte, [...].

(cfr. libro VII, p. 71; libro XII, p. 125; libro XX, p. 222)

M $\omega l$ ti cadean $\omega$ supini [> giù] de i l $\omega$ r cavalli Per le perc $\omega$ sse del feroce Achille.

Il, XI, 179-180

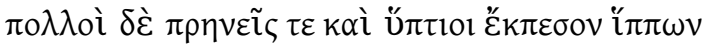

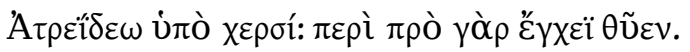

IL, Libro XXIII (p. 242)

Così Cosmondo in terra si distese

Per la percossa del feroce Achille. 


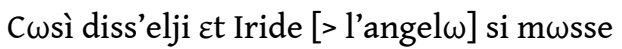

Per nwn difubidire ai suoi precetti.

Il, XI, 195

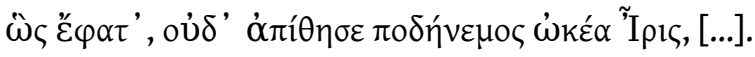

IL: -

L'hafta nutrita al vent $\omega$ havevaa in man $\omega$

Il, XI, 256

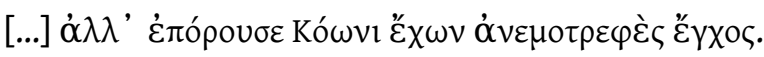

IL, Libro XII (p.122)

E così fieramente lo percosse

Con la dura asta sua nutrita al vento, [...].

IL, Libro XV (p. 161)

[...] Tutti con l'asta sua nutrita al vento.

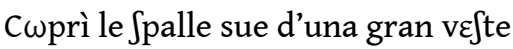

Di velut $\omega$ r $\omega \int$ at $\omega$ ad $\omega$ rna d'or $\omega$

Il: -

IL, Libro VI (p. 63)

E si vestì la bella cotta d'arme

Di veluto rosin cosperso d'oro, [...].

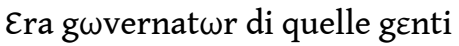

Reverito dal ${ }^{l \omega r} p \omega p \omega l$ c $\omega$ me ${ }^{\text {su } \omega}$ un Di $\omega$

Il, XI, 58

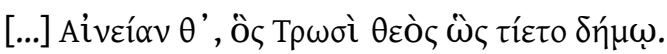

IL, Libro XV (p.159)

Siamo onorati ne le terre nostre,

Che ci aman con timor, come un lor Dio.

\section{NOTES}

1. Quondam parla dell' Italia liberata nei termini di «gigantesca macchina da riproduzione» dell'Iliade (A. QUONDAM, La poesia duplicata. Imitazione e scrittura nell'esperienza del Trissino, in N. POZZA [a cura di], Atti del Convegno di Studi su Giangiorgio Trissino, Vicenza, Accademia Olimpica, 31 marzo-1 aprile 1979, Vicenza, Neri Pozza, 1980, pp.67-109; p.90); Zatti definisce l'epica trissiniana un' «epica del "precetto"», che obbliga il lettore "a riattivare di volta in volta nella memoria l'ipotesto omerico [...], a mettere a nudo il sistema soggiacente delle equivalenze» (s. ZATTI, L'imperialismo epico del Trissino, in ID., L'ombra del Tasso. Epica e romanzo nel Cinquecento, Milano, Mondadori, 1996, pp. 56-110; pp. 103-104); Gigante, infine, parla di «"ansia" trissiniana di emulazione dell'archetipo» (c.GIGANTE, Un'interpretazione dell'Italia liberata dai Goti, in ID., Esperienze di filologia cinquecentesca. Salviati, Mazzoni, Trissino, Costo, Il Bargeo, Tasso, Roma, Salerno, 2003, pp. 46-79; p. 65. Prima in C. GIGANTE, «Azioni formidabili e misericordiose». L'esperimento epico del Trissino, «Filologia e Critica», XXIII, I, 1998, pp. 44-71).

2. Palese è l'ammirazione di Trissino nei confronti di Omero tanto nella lettera con cui il Vicentino dedica il suo poema all'imperatore Carlo V (G. G. TRISsINo, La Italia liberata da Gotti, in S. MAFFEI [a cura di], Tutte le opere di Giovan Giorgio Trissino, gentiluomo vicentino, non più raccolte, Verona, appresso Jacopo Vallarsi, vol. I, XxxVII-XLI), quanto all'interno della sua Poetica, in 
particolare della Divisione VI (G.G. TRISSINo, Poetica (V-VI), in B. WEINBERg [a cura di], Trattati di retorica e di poetica, vol. II, Bari, Laterza, pp. 5-90).

3. Tra questi si vedano almeno: G. BALDASSARRI, Il sonno di Zeus. Sperimentazione narrativa del poema rinascimentale e tradizione omerica, Roma, Bulzoni, 1982; S. ZATTI, L'imperialismo epico, cit.; C. GIGANTE, Un'interpretazione dell'Italia, cit.; e infine i recenti M. VITALE, L'Omerida italico: Gian Giorgio Trissino: appunti sulla lingua dell'Italia liberata da' Gotthi, Venezia, Istituto di Lettere Scienze ed Arti, Classe di scienze morali lettere ed arti, 2010 e F. DI SANTO, Il poema epico rinascimentale e l'Iliade: da Trissino a Tasso, Tesi di Dottorato in Memoria Culturale, Università di Pisa, A.A. 2011/2012, Relatore Prof. G. Paduano.

4. Ricerca che rientra nell'ambito di una più ampia indagine sul poema trissiniano, cui sto dedicando la mia tesi di dottorato.

5. Scrive Bernardo Morsolin, autore della monografia trissiniana tuttora più documentata ed esaustiva: «Le due ultime Divisioni della Poetica erano belle e compiute, quando il Trissino partiva alla volta di Germania», ovvero nell'aprile del 1550, data a cui risale la partenza del Vicentino per Augusta, nella vana speranza di essere ricevuto dall'Imperatore. Specifica ulteriormente lo storico ottocentesco: «Le due Divisioni della Poetica, quantunque compiute e intitolate al Perrenot a mezzo il 1550, non uscirono subito in luce. [...] Chi la pubblicò la prima volta fu Andrea Arrivabene, quando, morto da più che tredici anni l'autore, viveva ancora il Perrenot [...]», B. MORSoLIN, Giangiorgio Trissino. Monografia di un gentiluomo letterato del secolo XVI, Firenze, Le Monnier, 1894, p. 337-339.

6. TRISSINO, Poetica (V-VI), 46.

7. Si ricordi, in tal senso, il paradigma fissato da Baldassarri a proposito dell'imitatio omerica all'interno dei poemi narrativi rinascimentali, che prevede da un lato «una presenza sistematica e costante dell'Iliade», dall'altro «un'eclissi altrettanto sistematica di un poema come l'Odissea» (G. BALDASSARRI, Il sonno di Zeus, cit., p. 10). Gigante, nella lettura che dà del poema trissiniano, sfata parzialmente lo schema proposto da Baldassarri; scrive infatti che il Vicentino «nell'Italia liberata - che per ogni riguardo sembra costituire un caso a sé - non si limita all'imitazione in modo pedissequo e sostanzialmente equivoco [...] dell'Iliade, ma anche, sia pure occasionalmente, dell'Odissea». L'atteggiamento che Trissino rivela nei confronti dell'Omero dell'odissea - specifica lo studioso - è ben diverso da quello di altri autori rinascimentali che, altrettanto occasionalmente, hanno guardato a tale poema: «non si tratta per lui, come invece per Ariosto e poi per Tasso, di celebrare, attraverso il filtro dantesco, Odisseo come precursore "figurale" di Colombo e degli altri scopritori di nuove terre»; piuttosto «il mito di Odisseo è da Trissino rivissuto in un orizzonte "archeologico" lontano da ogni suggestione "moderna". Anche con l'odissea il suo rapporto è, in definitiva, ancora una volta, mimetico, di duplicazione» (C. GIGANTE, Un'interpretazione dell'Italia, cit., pp. 74-75). Che nell'opera trissiniana anche l'imitazione dell'altro poema omerico avvenga in termini di remake non c'è dubbio. Si tenga presente però che, quando Gigante parla di imitatio occasionale dell'Odissea, bisogna intendere le sue parole alla lettera: il confronto tra l'Italia e l'odissea - condotto all'interno della mia tesi dottorale, ma cui non si farà cenno all'interno dell'articolo - non ha permesso di individuare che pochissimi richiami diretti al secondo dei poemi di Omero, dei quali il più eclatante è certamente quello già discusso dallo stesso Gigante all'interno del suo saggio. Si tratta di passi di cui è doveroso dare conto, ma che, per la loro frequenza così rarefatta, non consentono di scostare di molto Trissino dal paradigma proposto da Baldassarri.

8. TRISSINo, Poetica (V-VI), 46.

9. Ivi, 50 .

10. TRISSINO, Poetica (V-VI), 46.

11. Senza contare l'appello proemiale «Divino Apollo, e voi celesti Muse», e l'invocazione che apre il ventisettesimo e ultimo libro dell'Italia liberata, «Vergini sacre al cui governo è posto/ 
Parnaso [...]», gli interventi diretti dell'autore all'interno del poema si riducono a nove: libro II, p. 13 e p. 14; libro VIII, p. 78; libro IX, p. 87; libro X, p. 101; libro XII, p. 128; libro XV, p. 161; libro XVIII, p. 189 e p. 191. (Le pagine citate, come avverrà in seguito, si riferiscono all'edizione del poema curata da Scipione Maffei nel 1729: TRISsINo, La Italia liberata, pp. 1-296). Tutti coincidono con preghiere alle Muse, che Trissino chiama in causa soprattutto nel corso delle azioni belliche, affinché gli forniscano l'elenco completo dei guerrieri uccisi da questo o da quel condottiero durante la battaglia.

12. TRISSINO, La Italia liberata, 13.

13. A proposito dell'uso della similitudine nell'Italia liberata, Sergio Zatti ne parla nei termini di «contrassegno» del testo come epico, rinvio costante al codice di riferimento, che è per l'appunto quello dell'epos. Nel poema trissiniano, scrive ancora Zatti, il meraviglioso romanzesco risulta bandito in ogni sua forma, anche per ciò che riguarda la retorica narrativa: da qui il sacrificio della metafora a favore della similitudine, che svolge sostanzialmente il ruolo della descrizione. Si veda s. ZATTI, L'imperialismo epico, ed. cit, p. 106-107.

14. Un'interessante lettura della lettera di dedica al «Clementissimo et Invittissimo Imperatore» Carlo V, accompagnata da un'acuta analisi delle differenze di posizione assunte da Trissino in tale lettera e nella Divisione VI della Poetica, si trova in E. MUSACCHIO, Lo stile del nuovo poema epico rinascimentale, «Letteratura Italiana Antica», VI, 2005, pp. 369-389.

15. TRISSINO, La Italia liberata, XXXIX.

16. Ibidem.

17. La carta $162 r$ del Fondo Castiglioni $8 / 1$ - manoscritto che, come si avrà l'opportunità di descrivere nel corpo del testo, contiene appunti di mano di Trissino in vista della stesura della sua opera magna - mostra, sulla colonna di destra, un indice preparatorio dell'Italia liberata, in cui il numero di ogni libro del poema è accompagnato da una breve descrizione del suo contenuto. La numerazione si ferma al libro ventiquattresimo, il cui argomento è abbozzato in questi termini: «24. Combatter di Vitige co(n) Belifario che lo prende poi pilja Ravenna $\varepsilon$ va a Bifanzo». In realtà, nell'edizione definitiva del poema, la cattura di Vitige, la liberazione definitiva dell'Italia dal giogo della schiavitù gota, e il rientro di Belisario e compagni a Bisanzio saranno oggetto di narrazione del ventisettesimo canto dell'Italia liberata. Da qui la supposizione che Trissino in un primo momento avesse pensato di strutturare l'opera in ventiquattro libri, come l'Iliade, per poi vedersi costretto ad ampliarla. Sulla mole del poema trissiniano e sul suo sbilanciamento interno - l'autore narra in più dieci libri (X-XXIV) l'assedio di Roma e dedica unicamente i libri XXV e XXVI al resoconto delle battaglie svoltesi in varie città dell'Italia centro-settentrionale - si veda C. GIGANTE, Un'interpretazione dell'Italia, cit., p. 52 e pp. 57-58.

18. All'interno dell'Italia liberata alle armi viene concesso molto più spazio che agli amori. Nello specifico, i libri occupati interamente o quasi da eventi bellici sono: libro VII (battaglia di Napoli); libro XII (battaglia di Ponte Mole); libro XV (assedio di Roma); libro XVII (duello AcquilinoTurrismondo); libro XVIII (assedio di Roma); libro XX (duello Achille-Argalto e lotta confusa); libro XXI (duello Corsamonte-Turrismondo e lotta confusa); libro XXII (gesta di Corsamonte e sua morte); libri XXV-XXVII (presa di Milano, Pavia, Osimo, Rimini, Ravenna e duello finale).

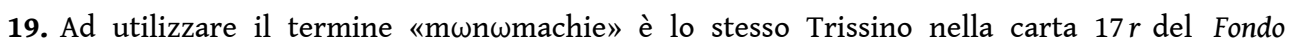
Castiglioni 8/1, in cui l'autore elenca i duelli, le «scaramuzze», i «fatti d'arme», gli amori e i tradimenti che ha intenzione di inserire nell'Italia liberata.

20. Nell'Italia liberata preparativi del tutto simili, per esempio, precedono il duello AcquilinoTurrismondo del libro XVII (p. 176): giuramenti, disegno del campo di battaglia, costruzione dei due opposti padiglioni dove i combattenti vanno ad armarsi. Nel caso del libro XxI, invece, il rituale risulta interrotto; Turrismondo si avvicina a Corsamonte chiedendo di giurare che il vincitore del duello restituisca il corpo del nemico vinto ai compagni, ma Corsamonte indignato risponde: «Crudel Baron, non mi parlar di patti,/ Avendo fatte a me sì gravi offese,/ [...] l'odio 
nostro non ricerca/ Patti, né tregue, che sariano indarno,/ Se l'un di noi non cade in sul terreno,/ E non dona col sangue a l'altro gloria» (p. 219). Si entra così immediatamente nel vivo del combattimento.

21. Iliade IV, 120. L'edizione dell'Iliade (Il. d'ora in poi) alla quale si è fatto riferimento è: OMERo, Iliade, a cura di M. G. CIANI, commento di E. AVEZZù, Marsilio, Venezia, 1990.

22. TRISSINO, La Italia liberata, p. 209.

23. Ivi, 210.

24. Ivi, 211.

25. Il. XX, 321

26. «Lo colpì con la lancia il divino Achille, alla testa; la testa si spaccò in due», Il. XX, 386-387.

27. «Non resistette l'elmo di bronzo e la punta dell'arma lo trapassò con impeto spezzando l'osso: si spappolò dentro tutto il cervello», Il. XX, 398-400.

28. «Lo colpì con la lancia nel dorso», Il. XX, 402.

29. La pietà nei confronti del nemico vinto è la caratteristica che più distingue l'eroe trissiniano da quello omerico: in più d'un'occasione Belisario, Corsamonte e compagni si lasciano commuovere da un goto supplicante per aver salva la vita, laddove invece i loro alter ego omerici si accaniscono sistematicamente contro i Troiani. Un altro esempio emblematico è rintracciabile nel libro xIII dell'Italia liberata, nel momento in cui Lucillo e Tibullo, poco prima della loro sortita notturna in campo goto, esaudiscono le preghiere di Frodino lasciandolo in vita, mentre invece, nel corrispondente episodio iliadico, Diomede ed Odisseo uccidono Dolone (libro x). Ancora più eclatante il caso di Corsamonte nel libro XXI che, uscito vincitore dal duello contro Turrismondo, acconsente a restituirne immediatamente il corpo ai nemici, quando invece è noto lo sconcio cui Achille destina il cadavere di Ettore prima che il troiano possa ricevere degno funerale e sepoltura. La pietà dei guerrieri trissiniani si spiega naturalmente alla luce della missione cristiana di cui si fanno portatori: non è un caso infatti che Trissino mostri propensi al perdono solo i Romani, per i quali parteggia apertamente, a differenza di Omero, autore super partes. A proposito di Belisario quale modello di miles Christi si veda: A. CORRIERI, Rivisitazioni cavalleresche ne L'Italia liberata da' Gotthi di Giangiorgio Trissino, «Schifanoia», XXXIV-XXXV, 2008, pp. 183-192.

30. «Figlio di Peleo, perché mi insegui con i tuoi piedi veloci? tu sei mortale e io immortale; non hai ancora capito che sono un dio e ti ostini nella tua furia [...] non potrai uccidermi, perché non sono mortale», Il. XXII, 8-10 e 13.

31. S. ZATTI, L'imperialismo epico..., cit., p. 71.

32. C. GIGANTE, Un'interpretazione..., cit., p. 78.

33. Ivi, 65 .

34. Celebre in tal senso il durissimo giudizio di Giraldi Cinzio nei confronti di Trissino all'interno del suo Discorso: «[...] come l'età di Omero e i costumi di que' tempi, e le singolari virtù che si trovano in questo divino poeta, fecero tollerabili quelle cose in lui, così ora ciò fare non sarebbe altro che voler scegliere dall'oro del suo componimento lo sterco [...] e pensare di averne scelto l'oro purissimo, come si può vedere nell'Italia del Trissino», (G. GIRALDI CINZIO, Discorso intorno al comporre de' romanzi, delle commedie, e delle tragedie, e di altre maniere di poesia, in ID., Scritti critici, a cura di C. GUERRIERI CROCETTI, Milano, Marzorati, 1973, p.63). A indignare tanto i critici rinascimentali erano in particolare due episodi dell'Italia, in cui traspare con evidenza come la volontà trissiniana di imitare pedissequamente Omero induca talvolta il poeta a rappresentare scene ai limiti del ridicolo o dell'indecoroso (si tratta di due episodi ascrivibili al gruppo di quegli «involontari ridenda» (C. GIGANTE, Un'interpretazione..., cit., p.69) che anche Benedetto Croce individuava nel poema, affermando "allieterebbero se il giuoco non fosse tirato troppo in lungo", cfr. B. CROCE, L'Italia liberata dai Goti, in ID., Poeti e scrittori del pieno e tardo Rinascimento, Bari, Laterza, 1945, vol. I, 302). Ci si riferisce nello specifico alla scena di seduzione di Giustiniano da parte di Teodora (libro III) - tutta modulata su quella di Zeus da parte di Era (Il., XIV) - durante la quale la 
regina sfrutta le sue arti femminili per convincere il marito a far rientrare Giustino dalla guerra. E alla vendetta che la Madonna, trasformata per l'occasione in una sorta di divinità omerica assetata di rivalsa, chiede a Dio nel libro X, pregandolo di punire Massenzo per lo stupro da lui compiuto in un luogo sacro durante l'assedio di Napoli. Sul primo dei due episodi si è già espresso anche Gigante, affermando: «La partecipazione della natura al regale "trastullarsi" [...] è spia di una delle ragioni per cui l'Italia liberata [...] fu sentita così fortemente estranea dal pubblico del secondo Cinquecento a cui si rivolgeva: la tecnica di rappresentazione è infatti priva del necessario "decoro", postulato come essenziale, soprattutto se associato, come in questo caso, a una "maestà", nella pubblicistica teorica di un Giraldi Cinzio, o, più tardi, ma sulla stessa falsariga, nei Discorsi dell'arte poetica di Torquato Tasso", C. GIGANTE, Un'interpretazione, cit., p. 73.

35. Ivi, p. 65.

36. Non autografa è unicamente la c. $28 r$, contenente un breve elenco di provincie e città della Grecia antica.

37. M. LIEBER, C. WEYERS, Giovan Giorgio Trissino: i manoscritti Castiglioni 8/1, 8/2, 8/3 della Biblioteca Braidense di Milano, in M. LIEBER, W. HIRDT (a cura di), Kunst und Kommunikation. Betrachtungenzum Medium Sprache in der Romania. Festschrift zum 60. Geburstag von Richard Baum, Tübingen, Stauffenburg, 1997, pp. 221-254. L'articolo in appendice riporta una tabella in cui il contenuto dei Fondi Castiglioni 8/1, 8/2, 8/3 è catalogato per carte, o per gruppi di carte, e brevemente descritto: ad essa si rimanda per avere un'idea più completa di cosa tali codici esattamente conservano e di come gli appunti trissiniani siano ordinati - se di ordine si può parlare - all'interno dell'8/1. Degli autografi trissiniani si è occupato anche Franco Tomasi in F. TOMASI, Gian Giorgio Trissino, in M. MOTOLESE, P. PROCACCIOLI, E. RUSSO (a cura di), Autografi dei letterati italiani. Il Cinquecento, vol. II, Roma, Salerno, 2013, pp. 369-385.

38. Degli studiosi che «a vario titolo e con possibilità di scavo differente» ebbero modo di mettere mano all'archivio privato della famiglia Trissino, presso cui, fino alla fine del XIX secolo, fu conservata la maggior parte dei testi autografi trissiniani, si è occupato Franco Tomasi in F. TOMASI, Gian Giorgio, cit., pp. 369-370.

39. Per approfondire la descrizione esterna completa del manoscritto si rimanda alla scheda online curata da Barbara Maria Scavo (http://manus.iccu.sbn.it//opac_SchedaScheda.php? ID=116646). La scheda si occupa dell'intero composito formato dai tre volumi Castiglioni 8/1-3, non descrivendo separatamente le unità codicologiche.

40. F. TOMASI, Gian Giorgio, cit., p. 370.

41. Più nello specifico, per quanto riguarda la colonna di destra, nel caso dei primi dodici libri dell'Iliade il verso iniziale è copiato per intero; per i rimanenti il Vicentino si limita ad annotare la prima parola o le prime due-tre parole del verso.

42. Una riflessione a parte, seppur breve, merita la scrittura trissiniana dello Zibaldone. Per l'analisi paleografica si rimanda a quella dettagliata fatta da Antonio Ciaralli in F. TOMASI, Gian Giorgio, cit., p. 376. Il fatto che in questa sede deve essere menzionato è che tutte le carte dello Zibaldone risultano vergate in una scrittura che segue le norme della seconda riforma ortografica trissiniana, elemento potenzialmente assai utile ai fini della datazione del manoscritto. Esso infatti consente di fissare un terminus post quem: il 1529, data a cui risale il secondo alfabeto del Vicentino (per approfondire sulle caratteristiche delle due riforme ortografiche trissiniane si veda: B. MIGLIORINI, Le proposte trissiniane di riforma ortografica, in «Lingua Nostra», XI, 4, 1950, pp. 77-81). Si tenga però presente che si ha a che fare con annotazioni personali dell'autore $\mathrm{e}-\mathrm{se}$ è vero che Trissino, anche nelle scritture più intime e private, non manca mai di conformarsi alla nuove regole ortografiche da lui proposte - è altrettanto vero che lo fa in maniera oscillante. Non mancano, insomma, incongruenze, sintomi del fatto che tale riforma di per sé stessa era destinata a causare non poca confusone perfino al suo stesso creatore e pressoché unico fautore.

43. TRISSINO, La Italia liberata, cit., p. 123. 
44. «Come quando lo sposo di Era dai bei capelli scaglia una folgore che annuncia un fitto rovescio di pioggia o grandine o neve - quando la neve copre la terra - oppure l'abisso immenso di una guerra crudele, così in fondo all'animo soffriva profondamente Agamennone e il cuore gli tremava nel petto. Quando volgeva gli occhi alla pianura di Troia, guardava sgomento i fuochi che davanti ad Ilio ardevano fitti, sgomento udiva il suono di flauti e zampogne, il clamore degli uomini; e quando guardava le navi e l'esercito acheo, si strappava i capelli dal capo invocando l'altissimo Zeus e profondamente soffriva nel cuore. Gli sembrò infine che l'idea migliore fosse di andare da Nestore figlio di Neleo, per studiare con lui un piano perfetto che a tutti i Danai potesse recare salvezza». Il. x, 5-20.

45. È lo stesso Belisario che, nel libro VI dell'Italia liberata - replicando a Magno e alle sue lodi indirizzate alla squadra di Pompeio per l'ordine con cui tali schiere sono abituate a marciare, perfino per andare a mensa - elenca le cinque principali doti di un buon comandante, fornendo in pratica un ritratto di sé stesso: «E poi, se ben la più onorevol cura/ Del capitanio è di nutrir le genti,/ Tal, che non manchi vittuaria al campo;/ E la seconda è di tenerle sane/ Con frequenti esercizi, e con fatiche;/ La terza è pur, che siano instrutte, e dotte/ Ne l'ordinanze, et arti de la guerra;/ Come la quarta è, ch'animose, e pronte/ Le facia a voler porsi entr'a i perigli;/ E poi la quinta è, ch'ubbidiscan tutte/ Al capo lor senza tardanza alcuna» (p. 55-56).

46. Nello specifico i passi dell'Iliade interessati sono: XI 298; XI 299-300; XI 297-298; XI, 291; XI, 292-295; XI, 177-178; XI 179-180; XI, 195; XI, 256; XI, 58. Per chiarezza si allega in appendice una tabella delle corrispondenze tra le prove versificatorie della c. $37^{1} v$ dello Zibaldone, la forma con cui tali versi eventualmente compaiono nella versione definitiva dell'Italia liberata, e i passi iliadici che fungono loro da modello.

47. Insieme ad esso, le altre prove di versi del foglio $37^{1} v$ che non risultano poi inserite nell'Italia

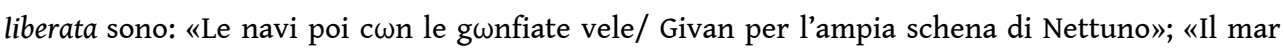

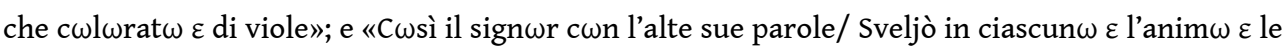
forze».

48. TRISSINO, La Italia liberata, cit., p. 158.

49. Da non escludere che i versi contenenti la descrizione dello scudo di Totila contengano anche una reminescenza dantesca. Si ricordi infatti che in Inf. VII, per descrivere il colore delle acque del «tristo ruscel» che scorre verso lo Stige, Dante usa l'aggettivo «persa» («L'acqua era buia assai più che persa», VII, 103). E nel medesimo canto, poco più sopra, aveva fatto riferimento a Cariddi all'interno di una celebre similitudine utile a descrivere la pena inflitta ad avari e prodighi: «Come fa l'onda là sovra Cariddi, / che si frange con quella in cui s'intoppa,/ così convien che qui la gente riddi» (VII, 22-24).

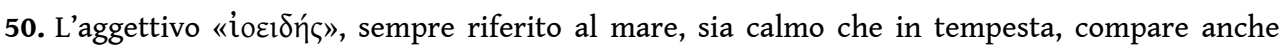
nell'Odissea, nei libri V (v. 56) e XI (v. 107); nell'Iliade invece ricorre solo nel passo citato nel corpo del testo.

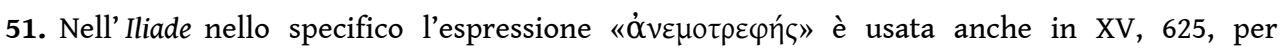
descrivere non più una lancia ma un'onda del mare $(\kappa \tilde{\nu} \mu \alpha)$ che il vento, appunto, 'nutre', ovvero solleva.

52. Sui tentativi di formularità trissiniana e sulla lingua dell'Italia modulata su quella omerica si vedano M. VITALE, L'Omerida italico e F. DI SANTO, Il poema epico, cit., pp. 281-296.

53. TRISSINO, La Italia liberata, cit., p. 122.

54. Ivi, 161.

55. Trissino aveva studiato greco a Milano, dove si era recato nel settembre del 1506 e dove dimorò circa due anni, sotto la guida di Demetrio Calcondila. Scrive Morsolin: «È dovuto in modo particolare alla fama e al valore singolare di Demetrio Calcondila, se il Trissino in un tempo, che l'Italia annoverava tra i più riputati ellenisti il Lascaris e il Musuro, preferì di recarsi a Milano", B. MoRsolin, Giangiorgio Trissino, cit., pp. 20-21. E ancora, circa le qualità del Vicentino come 
grecista: «In capo a un anno [...], il Trissino avea così profittato dei precetti del Calcondila, che, misuratosi con un greco in una quistione di prosodia, potè difendere la sua sentenza con copia di argomenti, tolti da Prisciano, da Diomede e dall'uso. [...], è anzi a pensare, ch'egli fosse riputato fin d'allora tra gli uomini più periti della lingua greca», Ivi, pp. 25-26.

\section{RÉSUMÉS}

L'articolo offre alcune riflessioni sulle caratteristiche dell'imitatio omerica trissiniana all'interno dell'Italia liberata dai Goti, poema eroico da sempre considerato una sorta di copia rinascimentale dell'Iliade. L'analisi di uno degli innumerevoli blocchi narrativi che il Vicentino mutua dall'rliade per innestarlo, con pochi indispensabili adattamenti, all'interno dell'Italia, è seguita da un'indagine condotta sullo Zibaldone dell'Italia liberata, manoscritto autografo contenente appunti e sperimentazioni di versi preparatori alla stesura del poema, alcune carte del quale sono considerabili alla stregua di un laboratorio di traduzione omerica.

L'article propose des réflexions sur l'imitatio homérique de Giangiorgio Trissino à l'intérieur de l'Italia liberata dai Goti, poème héroïque considéré depuis toujours comme une sorte de reproduction de l'Iliade à la Renaissance. L'analyse se concentre d'abord sur un des nombreux passages que le poète emprunte à l'Iliade pour l'insérer, avec des modifications nécessaires, à l'intérieur de son épopée. La recherche porte ensuite sur le Zibaldone dell'Italia liberata, le manuscrit autographe qui contient des notes et des essais des vers préparatoires à l'écriture du poème. Plusieurs de ces feuillets peuvent être considérés comme une sorte de laboratoire de traduction d'Homère.

\section{INDEX}

Index chronologique : XVIe siècle

Index géographique : domaine italien

Mots-clés : Omero, Giangiorgio Trissino, riscritture, imitazione, poema epico

\section{AUTEUR}

\section{PAOLA PECCI}

Paola Pecci si è laureata in Lettere (2009) e quindi specializzata in Filologia Moderna (2011) presso l'Università degli Studi di Padova. Nel 2012 è risultata vincitrice di una borsa presso la Scuola di Dottorato in Scienze Linguistiche, Filologiche e Letterarie dell'Università di Padova. Nel 2016 discuterà la sua tesi dottorale relativa all'Italia liberata dai Goti di Giangiorgio Trissino. 